\title{
Stabilization for two-dimensional delta operator systems with time-varying delays and actuator saturation
}

\author{
Dan Peng ${ }^{1 *}$, Ting Zhang ${ }^{1}$ and Hongjiu Yang ${ }^{2}$
}

\section{"Correspondence:}

dpeng1219@163.com

'School of Science, Yanshan

University, Qinhuangdao, China

Full list of author information is

available at the end of the article

\begin{abstract}
In this paper, stabilization is studied for a two-dimensional delta operator system with time-varying delays and actuator saturation. Both lower and upper bounds of the time-varying delays are considered. An estimate of the domain of attraction for the two-dimensional delta operator system is introduced to analyze stability of the closed-loop system. A state feedback controller is designed via a Lyapunov-Krasovskii functional approach for the two-dimensional delta operator system with time-varying delays and actuator saturation. Two numerical examples are given to illustrate the effectiveness and advantages of the developed techniques.
\end{abstract}

Keywords: Two-dimensional (2-D) systems; Delta operator systems; Actuator saturation; Time-varying delays

\section{Introduction}

A 2-D system is a dynamic process in which information is transmitted in two independent directions. 2-D systems are widely studied due to the fact that many practical systems are usually modeled as the 2-D systems, such as signal and image processing [1], thermal processing [2], metal rolling processing [3], and so on. Owing to the fact that 2-D systems have wide application background, stabilization analysis for the 2-D systems has become an important problem in control field. Stability, $l_{2}$-gain, and stabilization analysis have been investigated for 2-D discrete switched systems [4, 5]. Many practical 2-D systems contain inherent delays which are often sources of poor performances and instability for the 2-D systems. Therefore, considerable interests have been attracted in stabilization analysis for the 2-D systems with time delays. And stabilizations are studied mainly by delay-dependent methods for the 2-D systems with time delays, because the delay-dependent methods are less conservative than the delay-independent ones $[6,7]$. An $H_{\infty}$ control problem has been considered for a 2-D T-S fuzzy model with time delays and missing measurements [8]. A delay-dependent $H_{\infty}$ controller has been designed for a 2-D switched system with time delays [9]. For 2-D discrete-time systems with interval time-varying delays, a delay-dependent stability problem has been studied in [10]. Based on [10], an improved approach has been given in [11]. Besides, a delay-partitioning approach has been proposed in [12]. Most of the studies are mainly on 2-D discrete-time

(c) The Author(s) 2018. This article is distributed under the terms of the Creative Commons Attribution 4.0 International License (http://creativecommons.org/licenses/by/4.0/), which permits unrestricted use, distribution, and reproduction in any medium, provided you give appropriate credit to the original author(s) and the source, provide a link to the Creative Commons license, and indicate if changes were made. 
systems. Hence, there is a lot of space to extend the 2-D systems with time delays into delta domain, which motivates us to make an effort in this paper.

Discrete-time systems have been widely researched with the rapid development of computer technology. The discrete-time systems are usually expressed as shift operator systems. Studies on 2-D discrete-time systems are also mainly about 2-D shift operator systems. However, parameters in the traditional shift operator systems do not tend to the ones in corresponding continuous-time systems when sampling frequencies are gradually increased, which usually leads to poor performances and instability for the control systems [13]. In order to solve the problems, delta operators are proposed to replace the traditional shift operators in fast sampling cases [14]. After that, a lot of research results have been shown for delta operator systems. Using a delta operator approach, a fuzzy fault detection filter and a stability problem have been investigated for uncertain fuzzy and networked control systems, respectively $[15,16]$. Saturation is a common problem in modern engineering field. It is meaningful to research actuator saturation because most of actuators do not strictly accord with linearity and many of them subject to saturation in real physical systems. Recently, considerable interest has been attracted to analyze control systems subject to actuator saturation, please refer to [17-19] and the references therein. A convex hull approach has been proposed to deal with systems with actuator saturation [20-22]. Moreover, the domain of attraction is a subset of state space, and all system trajectories that start from the subset will eventually tend to origin. An estimate of the domain of attraction has been investigated for a class of nonlinear systems subject to input constraints [23, 24]. 2-D models widely exist in modern engineering field, such as a metal rolling process and a thermal process. However, the 2-D models are rarely considered for fast sampling cases in the existing results.

In this paper, a 2-D system is considered in delta domain to adapt to a fast sampling rate and avoid the system instability caused by the fast sampling in this paper. Moreover, both time-varying delays and actuator saturation, which usually occur in the modern engineering field, are studied for a 2-D delta operator system. Free weighting matrices, 2-D Jensen inequalities, and linear matrix inequalities (LMIs) approaches are applied to stabilization analysis. Furthermore, an estimate of the domain of attraction is proposed for the 2-D delta operator system. A state feedback controller is designed by Lyapunov-Krasovskii methods. Two numerical examples are shown to illustrate the effectiveness and advantages of the developed techniques.

This paper is organized in the following. Section 2 formulates problem formulation on the 2-D delta operator system with time-varying delays and actuator saturation. The stabilization problem is shown for the 2-D delta operator system with time-varying delays and actuator saturation in Sect. 3. In Sect. 4, two numerical examples are given to illustrate the effectiveness and advantages of the proposed methods. The paper is concluded in Sect. 5 .

Main novelties of this paper are summarized as follows:

(1) A stabilization problem on a 2-D system is extended to delta domain which is a link between $s$-domain and $z$-domain.

(2) 2-D models widely exist in modern engineering field. However, the 2-D models are rarely considered for fast sampling cases in the existing results. In this paper, the 2-D model is considered in the case of fast sampling.

(3) An estimate of the domain of attraction is proposed for the 2-D delta operator system in this paper. 
Notation In the sequel, if not explicitly stated, matrices are assumed to have compatible dimensions. Throughout this paper, $R^{n}$ denotes the $n$-dimensional Euclidean space. For any matrix $A, \lambda_{\max }(A)$ denotes the maximal module of its eigenvalues, $A^{T}$ denotes the transpose of matrix $A, A>0$ and $A \geq 0$ denote that matrix $A$ is a positive definite matrix and a semi-positive definite matrix, respectively. $I$ is the identity matrix of appropriate dimension. The shorthand $\operatorname{diag}\left\{M_{1}, M_{2}, \ldots, M_{r}\right\}$ denotes a block diagonal matrix with diagonal blocks being the matrices $M_{1}, M_{2}, \ldots, M_{r}$. The symmetric terms in a symmetric matrix are denoted by $* \frac{\partial x(s, t)}{\partial s}$ and $\frac{\partial x(s, t)}{\partial t}$ denote partial derivatives of function $x(s, t)$ to variables $s$ and $t$, respectively.

\section{Problem formulation}

In this paper, two 2-D delta operators are shown as follows:

$$
\delta^{v} x\left(t_{i}, t_{j}\right)= \begin{cases}\partial x\left(t_{i}, t_{j}\right) / \partial t_{j}, & \mathrm{~T}_{v}=0, \\ \frac{x\left(t_{i}, t_{j}+\mathrm{T}_{v}\right)-x\left(t_{i}, t_{j}\right)}{\mathrm{T}_{v}}, & \mathrm{~T}_{v} \neq 0\end{cases}
$$

and

$$
\delta^{h} x\left(t_{i}, t_{j}\right)= \begin{cases}\partial x\left(t_{i}, t_{j}\right) / \partial t_{i}, & \mathrm{~T}_{h}=0, \\ \frac{x\left(t_{i}+\mathrm{T}_{h}, t_{j}\right)-x\left(t_{i}, t_{j}\right)}{\mathrm{T}_{h}}, & \mathrm{~T}_{h} \neq 0\end{cases}
$$

where $\delta^{v} x\left(t_{i}, t_{j}\right)$ is the delta operator along vertical direction, $\delta^{h} x\left(t_{i}, t_{j}\right)$ is the delta operator along horizontal direction, $\mathrm{T}_{\nu}$ is the sampling period along vertical direction, $\mathrm{T}_{h}$ is the sampling period along horizontal direction, $j$ and $i$ are time steps with $t_{j}=j \mathrm{~T}_{v}$ and $t_{i}=i \mathrm{~T}_{h}$, respectively. A 2-D delta operator system with time-varying delays and actuator saturation is given as

$$
\begin{aligned}
\delta x\left(t_{i+1}, t_{j+1}\right)= & \bar{A}_{1} x\left(t_{i+1}, t_{j}\right)+\bar{A}_{1 d} x\left(t_{i+1}, t_{j}-d_{1}\left(t_{j}\right)\right)+\bar{B}_{1} \operatorname{sat}\left(u\left(t_{i+1}, t_{j}\right)\right) \\
& +\bar{A}_{2} x\left(t_{i}, t_{j+1}\right)+\bar{A}_{2 d} x\left(t_{i}-d_{2}\left(t_{i}\right), t_{j+1}\right)+\bar{B}_{2} \operatorname{sat}\left(u\left(t_{i}, t_{j+1}\right)\right),
\end{aligned}
$$

with

$$
\begin{aligned}
& \delta x\left(t_{i+1}, t_{j+1}\right)=\delta^{v} x\left(t_{i+1}, t_{j}\right)+\delta^{h} x\left(t_{i}, t_{j+1}\right), \\
& \delta^{v} x\left(t_{i+1}, t_{j}\right)=\bar{A}_{1} x\left(t_{i+1}, t_{j}\right)+\bar{A}_{1 d} x\left(t_{i+1}, t_{j}-d_{1}\left(t_{j}\right)\right)+\bar{B}_{1} \operatorname{sat}\left(u\left(t_{i+1}, t_{j}\right)\right), \\
& \delta^{h} x\left(t_{i}, t_{j+1}\right)=\bar{A}_{2} x\left(t_{i}, t_{j+1}\right)+\bar{A}_{2 d} x\left(t_{i}-d_{2}\left(t_{i}\right), t_{j+1}\right)+\bar{B}_{2} \operatorname{sat}\left(u\left(t_{i}, t_{j+1}\right)\right), \\
& \bar{A}_{1}=\frac{2 A_{1}-I}{\mathrm{~T}_{v}}, \quad \bar{A}_{1 d}=\frac{2 A_{1 d}}{\mathrm{~T}_{v}}, \quad \bar{B}_{1}=\frac{2 B_{1}}{\mathrm{~T}_{v}}, \\
& \bar{A}_{2}=\frac{2 A_{2}-I}{\mathrm{~T}_{h}}, \quad \bar{A}_{2 d}=\frac{2 A_{2 d}}{\mathrm{~T}_{h}}, \quad \bar{B}_{2}=\frac{2 B_{2}}{\mathrm{~T}_{h}},
\end{aligned}
$$

where $x\left(t_{i}, t_{j}\right) \in R^{n}$ is the plant state, $u\left(t_{i}, t_{j}\right) \in R^{m}$ is the control input, $A_{1}, A_{2}, A_{1 d}, A_{2 d}, B_{1}$, and $B_{2}$ are parameter matrices with appropriate dimensions. Note that $d_{1}\left(t_{j}\right)$ and $d_{2}\left(t_{i}\right)$ are time-varying delays along vertical direction and horizontal direction, respectively. $d_{1}\left(t_{j}\right)$ and $d_{2}\left(t_{i}\right)$ are satisfied with

$$
0<d_{1 m} \leq d_{1}\left(t_{j}\right) \leq d_{1 M}, \quad 0<d_{2 m} \leq d_{2}\left(t_{i}\right) \leq d_{2 M},
$$


where $d_{1 m}, d_{1 M}, d_{2 m}$, and $d_{2 M}$ are positive real numbers. The function "sat" is the standard saturation function with appropriate dimension. The saturation function is defined as

$$
\operatorname{sat}(u)=\left[\operatorname{sat}\left(u_{1}\right), \operatorname{sat}\left(u_{2}\right), \ldots, \operatorname{sat}\left(u_{m}\right)\right]^{T},
$$

where $\operatorname{sat}\left(u_{i}\right)=\operatorname{sgn}\left(u_{i}\right) \min \left\{1,\left|u_{i}\right|\right\}$.

Initial conditions are given as

$$
\left\{\begin{array}{l}
x\left(t_{i}, t_{j}\right)=\phi_{v}, \quad \forall 0 \leq t_{i} \leq z_{1}, t_{j}=-d_{1 M},-d_{1 M}+1, \ldots, 0, \\
x\left(t_{i}, t_{j}\right)=\phi_{h}, \quad \forall 0 \leq t_{j} \leq z_{2}, t_{i}=-d_{2 M},-d_{2 M}+1, \ldots, 0, \\
x\left(t_{i}, t_{j}\right)=0, \quad \forall t_{i}>z_{1}, t_{j}=-d_{1 M},-d_{1 M}+1, \ldots, 0 \\
x\left(t_{i}, t_{j}\right)=0, \quad \forall t_{j}>z_{2}, t_{i}=-d_{2 M},-d_{2 M}+1, \ldots, 0
\end{array}\right.
$$

where $\phi_{v}$ and $\phi_{h}$ are given vectors, $z_{1}$ and $z_{2}$ are any large positive integers.

The objective of this paper is to stabilize the 2-D delta operator system (3) under the following state feedback controller:

$$
u\left(t_{i}, t_{j}\right)=K x\left(t_{i}, t_{j}\right)
$$

By controller (6), the 2-D delta operator system (3) is transformed as follows:

$$
\begin{aligned}
\delta x\left(t_{i+1}, t_{j+1}\right)= & \bar{A}_{1} x\left(t_{i+1}, t_{j}\right)+\bar{A}_{1 d} x\left(t_{i+1}, t_{j}-d_{1}\left(t_{j}\right)\right)+\bar{B}_{1} \operatorname{sat}\left(K x\left(t_{i+1}, t_{j}\right)\right) \\
& +\bar{A}_{2} x\left(t_{i}, t_{j+1}\right)+\bar{A}_{2 d} x\left(t_{i}-d_{2}\left(t_{i}\right), t_{j+1}\right)+\bar{B}_{2} \operatorname{sat}\left(K x\left(t_{i}, t_{j+1}\right)\right)
\end{aligned}
$$

where $K \in R^{m \times n}$ is a feedback gain matrix.

The following definition on the domain of attraction for the 2-D delta operator system (3) will be used in this paper.

Definition 1 Denote a solution of system (3) with initial conditions in (5) as $\zeta\left(t_{i}, t_{j}, \phi_{v}, \phi_{h}\right)$. The domain of attraction for system (3) is given as

$$
\mathcal{T}:=\left\{\phi_{\nu} \in C_{1}\left[-d_{1 M}, 0\right], \phi_{h} \in C_{2}\left[-d_{2 M}, 0\right]: \lim _{t_{i}+t_{j} \rightarrow \infty} \zeta\left(t_{i}, t_{j}, \phi_{\nu}, \phi_{h}\right)=0\right\},
$$

where $\phi_{v}$ and $\phi_{h}$ are initial conditions, $C_{1}\left[-d_{1 M}, 0\right]$ is a set of the initial conditions $\phi_{v}$ with $0 \leq t_{i} \leq z_{1}, t_{j}=-d_{1 M},-d_{1 M}+1, \ldots, 0$ and $C_{2}\left[-d_{2 M}, 0\right]$ is a set of the initial conditions $\phi_{h}$ with $0 \leq t_{j} \leq z_{2}, t_{i}=-d_{2 M},-d_{2 M}+1, \ldots, 0$.

Based on Definition 1, an estimate of the domain of attraction is shown as follows:

$$
\begin{aligned}
\mathcal{Y}:= & \left\{\phi_{\nu} \in C_{1}\left[-d_{1 M}, 0\right], \phi_{h} \in C_{2}\left[-d_{2 M}, 0\right]:\right. \\
& \left.\max \left\|\phi_{v}\right\| \leq \eta_{1}, \max \left\|\phi_{h}\right\| \leq \eta_{2}, \max \left\|\delta \phi_{\nu}\right\| \leq \eta_{3}, \max \left\|\delta \phi_{h}\right\| \leq \eta_{4}\right\},
\end{aligned}
$$

where $\eta_{l}>0, l=1,2,3,4$, are the maximum positive scalars, $\|\cdot\|$ denotes the Euclidean norm. 
For a positive definite matrix $P \in R^{n \times n}$, an ellipsoid $\Omega(P)$ is defined as

$$
\Omega(P):=\left\{x\left(t_{i}, t_{j}\right) \in R^{n}: x^{T}\left(t_{i}, t_{j}\right) P x\left(t_{i}, t_{j}\right) \leq 1\right\} .
$$

For a matrix $H \in R^{m \times n}$, a linear region of saturation is given as

$$
\mathcal{L}(H):=\left\{x\left(t_{i}, t_{j}\right) \in R^{n}:\left|h_{q} x\left(t_{i}, t_{j}\right)\right| \leq 1, q=1,2, \ldots, m\right\}
$$

where $h_{q}$ is the $q$ th row of $H$.

Let $\mathcal{D}$ be a set of $m \times m$ diagonal matrices whose diagonal elements are either 1 or 0 . In the set $\mathcal{D}$, each element is labeled as $D_{p}, p=1,2, \ldots, 2^{m}$. Denote $D_{p}^{-}$as $D_{p}^{-}=I-D_{p}$. Note that $D_{p}^{-}$is also an element of the set $\mathcal{D}$.

Before ending this section, the following lemmas are given to develop the main results in this paper.

Lemma 1 ([25]) Let $K \in R^{m \times n}$ and $H \in R^{m \times n}$ be two given matrices. If $x\left(t_{i}, t_{j}\right) \in \mathcal{L}(H)$, then it is obtained that

$$
\operatorname{sat}\left(K x\left(t_{i}, t_{j}\right)\right) \in \operatorname{co}\left\{\left(D_{p} K+D_{p}^{-} H\right) x\left(t_{i}, t_{j}\right): p \in\left[1,2^{m}\right]\right\}
$$

where $\operatorname{co}\{\cdot\}$ stands for a convex hull. Consequently, $\operatorname{sat}\left(K x\left(t_{i}, t_{j}\right)\right)$ is rewritten as

$$
\operatorname{sat}\left(K x\left(t_{i}, t_{j}\right)\right)=\sum_{p=1}^{2^{m}} \eta_{p}\left(D_{p} K+D_{p}^{-} H\right) x\left(t_{i}, t_{j}\right)
$$

where $0 \leq \eta_{p} \leq 1$ and $\sum_{p=1}^{2^{m}} \eta_{p}=1$

Lemma 2 ([11]) For a matrix $W \in R^{m \times m}$ and a function $\omega\left(t_{i}, t_{j}\right) \in R^{m \times m}$, there exist

$$
\begin{aligned}
& \left(l_{2}-l_{1}+1\right) \sum_{t_{i}=l_{1}}^{l_{2}} \omega^{T}\left(t_{i}, t_{j}\right) W \omega\left(t_{i}, t_{j}\right) \geq\left(\sum_{t_{i}=l_{1}}^{l_{2}} \omega\left(t_{i}, t_{j}\right)\right)^{T} W\left(\sum_{t_{i}=l_{1}}^{l_{2}} \omega\left(t_{i}, t_{j}\right)\right), \\
& \left(l_{2}-l_{1}+1\right) \sum_{t_{j}=l_{1}}^{l_{2}} \omega^{T}\left(t_{i}, t_{j}\right) W \omega\left(t_{i}, t_{j}\right) \geq\left(\sum_{t_{j}=l_{1}}^{l_{2}} \omega\left(t_{i}, t_{j}\right)\right)^{T} W\left(\sum_{t_{j}=l_{1}}^{l_{2}} \omega\left(t_{i}, t_{j}\right)\right),
\end{aligned}
$$

where $W=W^{T}$ is a positive define matrix, $l_{1}$ and $l_{2}$ are integers satisfying $l_{1}<l_{2}$. Inequalities (8) and (9) are called 2-D Jensen inequalities.

Lemma 3 ([26]) Let $X, Y, \Psi, \Psi_{11}, \Psi_{12}, \Psi_{21}, \Psi_{22}, Z_{1}>0$, and $Z_{2}>0$ be given matrices with appropriate dimensions. The following two inequalities

$$
\left[\begin{array}{ccc}
-Z_{1} & \Psi_{11} & X \\
* & \Psi & \Psi_{12}^{T} \\
* & * & -Z_{1}
\end{array}\right]<0
$$


and

$$
\left[\begin{array}{ccc}
-Z_{2} & \Psi_{21} & Y \\
* & \Psi & \Psi_{22}^{T} \\
* & * & -Z_{2}
\end{array}\right]<0
$$

hold if and only if

$$
\left[\begin{array}{cc}
\Psi & \Psi_{11} \\
\Psi_{11}^{T} & -Z_{1}
\end{array}\right]<0, \quad\left[\begin{array}{cc}
\Psi & \Psi_{12} \\
\Psi_{12}^{T} & -Z_{1}
\end{array}\right]<0
$$

and

$$
\left[\begin{array}{cc}
\Psi & \Psi_{21} \\
\Psi_{21}^{T} & -Z_{2}
\end{array}\right]<0, \quad\left[\begin{array}{cc}
\Psi & \Psi_{22} \\
\Psi_{22}^{T} & -Z_{2}
\end{array}\right]<0,
$$

respectively.

Remark 1 In $[15,16]$, a delta operator is defined as the following form:

$$
\delta x\left(t_{k}\right)= \begin{cases}d x(t) / d t, & \mathrm{~T}=0, \\ \frac{x\left(t_{k}+\mathrm{T}\right)-x\left(t_{k}\right)}{\mathrm{T}}, & \mathrm{T} \neq 0 .\end{cases}
$$

In this paper, the delta operator is divided into two cases. On the one hand, a delta operator system is equivalent to a continuous-time system when the sampling period $\mathrm{T}$ equals zero. On the other hand, the delta operator system is equivalent to a discrete-time system when the sampling period $\mathrm{T}$ equals one. The delta operator system is a link between the continuous-time system and the discrete-time system. In this paper, the delta operator is extended to a 2-D model and the 2-D delta operators (1)-(2) are given for the 2-D delta operator system (3).

Remark 2 Compared with traditional shift operators, the 2-D delta operators (1)-(2) have obvious numerical advantages in fast sampling cases. According to the 2-D delta operators (1)-(2), a FM second system in delta domain is represented as the 2-D delta operator system (3). Note that the 2-D delta operator system (3) can be simplified into a common FM second system when the sampling period $\mathrm{T}$ equals one.

\section{Main results}

\subsection{Stability analysis}

In the subsection, a sufficient stability condition is provided for the 2-D delta operator system (3) with zero input.

Theorem 1 For given scalars $\tau_{1}, \tau_{2}, \tau_{3}, \tau_{4}, \tau_{5}, d_{1 m}, d_{2 m}, d_{1 M}$, and $d_{2 M}$, the 2-D delta operator system (3) with $u\left(t_{i}, t_{j}\right)=0$ is asymptotically stable if there exist matrices $X, Y, P>0$, $Q>0, R_{1}>0, R_{2}>0, Q_{1}>0, Q_{2}>0, S_{1}>0, S_{2}>0, Z_{1}>0, Z_{2}>0, M_{11}, M_{12}, M_{21}, M_{22}, N_{11}$, 
$N_{12}, N_{21}$, and $N_{22}$ such that the following LMIs hold:

$$
\left[\begin{array}{cccc}
-Z_{1} & \Psi_{11} & X & 0 \\
* & \Psi_{1} & \Psi_{12}^{T} & \Phi^{T} \\
* & * & -Z_{1} & 0 \\
* & * & * & \Psi_{2}
\end{array}\right]<0
$$

and

$$
\left[\begin{array}{cccc}
-Z_{2} & \Psi_{21} & Y & 0 \\
* & \Psi_{1} & \Psi_{22}^{T} & \Phi^{T} \\
* & * & -Z_{2} & 0 \\
* & * & * & \Psi_{2}
\end{array}\right]<0
$$

where

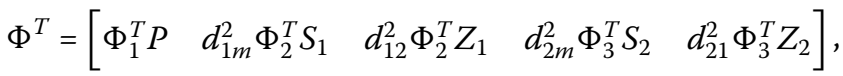

$$
\begin{aligned}
& \Psi_{11}=\left[\begin{array}{llllllll}
0 & 0 & M_{11} & 0 & M_{12} & 0 & 0 & 0
\end{array}\right] \text {, } \\
& \Psi_{12}=\left[\begin{array}{llllllll}
0 & 0 & N_{11} & 0 & N_{12} & 0 & 0 & 0
\end{array}\right] \text {, } \\
& \Psi_{21}=\left[\begin{array}{llllllll}
0 & 0 & 0 & M_{21} & 0 & M_{22} & 0 & 0
\end{array}\right] \text {, } \\
& \Psi_{22}=\left[\begin{array}{llllllll}
0 & 0 & 0 & N_{21} & 0 & N_{22} & 0 & 0
\end{array}\right] \text {, } \\
& \Psi_{1}=\left[\begin{array}{cccc}
-\bar{Q}+R+\hat{Q}-\bar{S} & 0 & \bar{S} & 0 \\
* & -\tilde{Q}+\bar{M}_{1}+\bar{M}_{1}{ }^{T}-\bar{N}_{1}-\bar{N}_{1}{ }^{T} & \bar{N}_{1}+\bar{M}_{2}{ }^{T}-\bar{N}_{2}{ }^{T} & -\bar{M}_{1} \\
* & * & -\bar{S}+\bar{N}_{2}+\bar{N}_{2}{ }^{T} & -\bar{M}_{2} \\
* & * & * & -R
\end{array}\right], \\
& \Psi_{2}=\operatorname{diag}\left\{-P,-d_{1 m}^{2} S_{1},-d_{12}^{2} Z_{1},-d_{2 m}^{2} S_{2},-d_{21}^{2} Z_{2}\right\}, \\
& \bar{Q}=\operatorname{diag}\{Q, P-Q\}, \quad R=\operatorname{diag}\left\{R_{1}, R_{2}\right\}, \\
& \Phi_{1}=\left[\begin{array}{llllllll}
\frac{1}{2}\left(\mathrm{~T}_{v} \bar{A}_{1}+I\right) & \frac{1}{2}\left(\mathrm{~T}_{h} \bar{A}_{2}+I\right) & \frac{1}{2} \mathrm{~T}_{v} \bar{A}_{1 d} & \frac{1}{2} \mathrm{~T}_{h} \bar{A}_{2 d} & 0 & 0 & 0 & 0
\end{array}\right], \\
& \tilde{Q}=\operatorname{diag}\left\{\mathrm{T}_{v} Q_{1}, \mathrm{~T}_{h} Q_{2}\right\} \text {, } \\
& \Phi_{2}=\left[\begin{array}{llllllll}
\frac{1}{2}\left(\mathrm{~T}_{v} \bar{A}_{1}-I\right) & \frac{1}{2}\left(\mathrm{~T}_{h} \bar{A}_{2}+I\right) & \frac{1}{2} \mathrm{~T}_{v} \bar{A}_{1 d} & \frac{1}{2} \mathrm{~T}_{h} \bar{A}_{2 d} & 0 & 0 & 0 & 0
\end{array}\right], \\
& \bar{S}=\operatorname{diag}\left\{\mathrm{T}_{v}^{2} S_{1}, \mathrm{~T}_{h}^{2} S_{2}\right\} \text {, } \\
& \Phi_{3}=\left[\begin{array}{llllllll}
\frac{1}{2}\left(\mathrm{~T}_{v} \bar{A}_{1}+I\right) & \frac{1}{2}\left(\mathrm{~T}_{h} \bar{A}_{2}-I\right) & \frac{1}{2} \mathrm{~T}_{v} \bar{A}_{1 d} & \frac{1}{2} \mathrm{~T}_{h} \bar{A}_{2 d} & 0 & 0 & 0 & 0
\end{array}\right], \\
& S_{1}=\tau_{1} P, \quad S_{2}=\tau_{2} P, \\
& Z_{1}=\tau_{3} P, \quad \bar{M}_{2}=\operatorname{diag}\left\{\mathrm{T}_{v} M_{12}, \mathrm{~T}_{h} M_{22}\right\}, \\
& \bar{N}_{1}=\operatorname{diag}\left\{\mathrm{T}_{v} N_{11}, \mathrm{~T}_{h} N_{21}\right\}, \quad \bar{N}_{2}=\operatorname{diag}\left\{\mathrm{T}_{v} N_{12}, \mathrm{~T}_{h} N_{22}\right\}, \quad Z_{2}=\tau_{4} P, \\
& Q=\tau_{5} P, \quad \hat{Q}=\operatorname{diag}\left\{\left(d_{12}+\mathrm{T}_{v}\right) Q_{1},\left(d_{21}+\mathrm{T}_{h}\right) Q_{2}\right\}, \quad \bar{M}_{1}=\operatorname{diag}\left\{\mathrm{T}_{v} M_{11}, \mathrm{~T}_{h} M_{21}\right\} .
\end{aligned}
$$


Proof Denote a Lyapunov-Krasovskii functional of the 2-D delta operator system (3) as

$$
V\left(x\left(t_{i}, t_{j}\right)\right)=V_{1}\left(x\left(t_{i}, t_{j}\right)\right)+V_{2}\left(x\left(t_{i}, t_{j}\right)\right),
$$

with

$$
\begin{aligned}
V_{1}\left(x\left(t_{i}, t_{j}\right)\right)= & \mathrm{T}_{v} x^{T}\left(t_{i+1}, t_{j+1}\right) Q x\left(t_{i+1}, t_{j+1}\right) \\
& +\mathrm{T}_{v} \sum_{\alpha=1}^{n_{1 M}} x^{T}\left(t_{i+1}, t_{j}-(\alpha-1) \mathrm{T}_{v}\right) R_{1} x\left(t_{i+1}, t_{j}-(\alpha-1) \mathrm{T}_{v}\right) \\
& +\mathrm{T}_{v}^{2} \sum_{\beta=n_{1 m}}^{n_{1 M}} \sum_{\alpha=1}^{\beta} x^{T}\left(t_{i+1}, t_{j}-(\alpha-1) \mathrm{T}_{v}\right) Q_{1} x\left(t_{i+1}, t_{j}-(\alpha-1) \mathrm{T}_{v}\right) \\
& +\mathrm{T}_{v}^{2} d_{1 m} \sum_{\beta=1}^{n_{1 m}} \sum_{\alpha=1}^{\beta} \bar{y}^{T}\left(t_{i+1}, t_{j}-(\alpha-1) \mathrm{T}_{v}\right) S_{1} \bar{y}\left(t_{i+1}, t_{j}-(\alpha-1) \mathrm{T}_{v}\right) \\
& +\mathrm{T}_{v}^{2} d_{12} \sum_{\beta=n_{1 m}+1}^{n_{1 M}} \sum_{\alpha=1}^{\beta} \bar{y}^{T}\left(t_{i+1}, t_{j}-(\alpha-1) \mathrm{T}_{v}\right) Z_{1} \bar{y}\left(t_{i+1}, t_{j}-(\alpha-1) \mathrm{T}_{v}\right), \\
V_{2}\left(x\left(t_{i}, t_{j}\right)\right)= & \mathrm{T}_{h} x^{T}\left(t_{i+1}, t_{j+1}\right)(P-Q) x\left(t_{i+1}, t_{j+1}\right) \\
& +\mathrm{T}_{h} \sum_{\alpha=1}^{n_{2 M}} x^{T}\left(t_{i}-(\alpha-1) \mathrm{T}_{h}, t_{j+1}\right) R_{2} x\left(t_{i}-(\alpha-1) \mathrm{T}_{h}, t_{j+1}\right) \\
& +\mathrm{T}_{h}^{2} \sum_{\beta=n_{2 m}}^{n_{2 M}} \sum_{\alpha=1}^{\beta} x^{T}\left(t_{i}-(\alpha-1) \mathrm{T}_{h}, t_{j+1}\right) Q_{2} x\left(t_{i}-(\alpha-1) \mathrm{T}_{h}, t_{j+1}\right) \\
& +\mathrm{T}_{h}^{2} d_{2 m} \sum_{\beta=1}^{n_{2 m}} \sum_{\alpha=1}^{\beta} \bar{y}^{T}\left(t_{i}-(\alpha-1) \mathrm{T}_{h}, t_{j+1}\right) S_{2} \bar{y}\left(t_{i}-(\alpha-1) \mathrm{T}_{h}, t_{j+1}\right) \\
& +\mathrm{T}_{h}^{2} d_{21} \sum_{\beta=n_{2 m}+1}^{n_{2 M}} \sum_{\alpha=1}^{\beta} \bar{y}^{T}\left(t_{i}-(\alpha-1) \mathrm{T}_{h}, t_{j+1}\right) Z_{2} \bar{y}\left(t_{i}-(\alpha-1) \mathrm{T}_{h}, t_{j+1}\right),
\end{aligned}
$$

where

$$
\begin{aligned}
& \bar{y}\left(t_{i+1}, t_{j}-\alpha \mathrm{T}_{v}\right)=x\left(t_{i+1}, t_{j}-(\alpha-1) \mathrm{T}_{v}\right)-x\left(t_{i+1}, t_{j}-\alpha \mathrm{T}_{v}\right), \\
& \bar{y}\left(t_{i}-\alpha \mathrm{T}_{h}, t_{j+1}\right)=x\left(t_{i}-(\alpha-1) \mathrm{T}_{h}, t_{j+1}\right)-x\left(t_{i}-\alpha \mathrm{T}_{h}, t_{j+1}\right) .
\end{aligned}
$$

Taking the delta operator manipulation of $V\left(x\left(t_{i}, t_{j}\right)\right)$, it is obtained that

$$
\delta V\left(x\left(t_{i}, t_{j}\right)\right)=\delta^{\nu} V_{1}\left(x\left(t_{i}, t_{j}\right)\right)+\delta^{h} V_{2}\left(x\left(t_{i}, t_{j}\right)\right),
$$

where

$$
\begin{aligned}
\delta^{v} V_{1}\left(x\left(t_{i}, t_{j}\right)\right) \\
=\xi_{1}^{T} \Phi_{1}^{T} Q \Phi_{1} \xi_{1}-x^{T}\left(t_{i+1}, t_{j}\right) Q x\left(t_{i+1}, t_{j}\right) \\
\quad+x^{T}\left(t_{i+1}, t_{j}\right) R_{1} x\left(t_{i+1}, t_{j}\right)-x^{T}\left(t_{i+1}, t_{j}-d_{1 M}\right) R_{1} x\left(t_{i+1}, t_{j}-d_{1 M}\right)
\end{aligned}
$$




$$
\begin{aligned}
& +\left(d_{12}+\mathrm{T}_{v}\right) x^{T}\left(t_{i+1}, t_{j}\right) Q_{1} x\left(t_{i+1}, t_{j}\right)-\mathrm{T}_{\nu} \sum_{\alpha=n_{1 m}}^{n_{1 M}} x^{T}\left(t_{i+1}, t_{j}-\alpha \mathrm{T}_{v}\right) Q_{1} x\left(t_{i+1}, t_{j}-\alpha \mathrm{T}_{v}\right) \\
& +d_{1 m}^{2} \bar{y}^{T}\left(t_{i+1}, t_{j}\right) S_{1} \bar{y}\left(t_{i+1}, t_{j}\right)-\mathrm{T}_{\nu} d_{1 m} \sum_{\alpha=1}^{n_{1 m}} \bar{y}^{T}\left(t_{i+1}, t_{j}-\alpha \mathrm{T}_{v}\right) S_{1} \bar{y}\left(t_{i+1}, t_{j}-\alpha \mathrm{T}_{v}\right) \\
& +d_{12}^{2} \bar{y}^{T}\left(t_{i+1}, t_{j}\right) Z_{1} \bar{y}\left(t_{i+1}, t_{j}\right)-\mathrm{T}_{v} d_{12} \sum_{\beta=n_{1 m}+1}^{n_{1 M}} \bar{y}^{T}\left(t_{i+1}, t_{j}-\beta \mathrm{T}_{v}\right) Z_{1} \bar{y}\left(t_{i+1}, t_{j}-\beta \mathrm{T}_{v}\right), \\
\delta^{h} V_{2}\left(x\left(t_{i}, t_{j}\right)\right) & \\
= & \xi_{1}^{T} \Phi_{1}^{T}(P-Q) \Phi_{1} \xi_{1}-x^{T}\left(t_{i}, t_{j+1}\right)(P-Q) x\left(t_{i}, t_{j+1}\right) \\
& +x^{T}\left(t_{i}, t_{j+1}\right) R_{2} x\left(t_{i}, t_{j+1}\right)-x^{T}\left(t_{i}-d_{2 M}, t_{j+1}\right) R_{2} x\left(t_{i}-d_{2 M}, t_{j+1}\right) \\
& +\left(d_{21}+\mathrm{T}_{h}\right) x^{T}\left(t_{i}, t_{j+1}\right) Q_{2} x\left(t_{i}, t_{j+1}\right)-\mathrm{T}_{h} \sum_{\alpha=n_{2 m}}^{n_{2 M}} x^{T}\left(t_{i}-\alpha \mathrm{T}_{h}, t_{j+1}\right) Q_{2} x\left(t_{i}-\alpha \mathrm{T}_{h}, t_{j+1}\right) \\
& +d_{2 m}^{2} \bar{y}^{T}\left(t_{i}, t_{j+1}\right) S_{2} \bar{y}\left(t_{i}, t_{j+1}\right)-\mathrm{T}_{h} d_{2 m} \sum_{\alpha=1}^{n_{2 m}} \bar{y}^{T}\left(t_{i}-\alpha \mathrm{T}_{h}, t_{j+1}\right) S_{2} \bar{y}\left(t_{i}-\alpha \mathrm{T}_{h}, t_{j+1}\right) \\
& +d_{21}^{2} \bar{y}^{T}\left(t_{i}, t_{j+1}\right) Z_{2} \bar{y}\left(t_{i}, t_{j+1}\right)-\mathrm{T}_{h} d_{21} \sum_{\beta=n_{2 m}+1}^{n_{2 M}} \bar{y}^{T}\left(t_{i}-\beta \mathrm{T}_{h}, t_{j+1}\right) Z_{2} \bar{y}\left(t_{i}-\beta \mathrm{T}_{h}, t_{j+1}\right),
\end{aligned}
$$

with

$$
\begin{aligned}
& \xi_{1}=\left[\begin{array}{llll}
x^{T} & x_{d}^{T} & x_{d m}^{T} & x_{d M}^{T}
\end{array}\right]^{T}, \\
& x=\left[\begin{array}{ll}
x^{T}\left(t_{i+1}, t_{j}\right) & x^{T}\left(t_{i}, t_{j+1}\right)
\end{array}\right]^{T}, \\
& x_{d}=\left[\begin{array}{ll}
x^{T}\left(t_{i+1}, t_{j}-d_{1}\left(t_{j}\right)\right) & x^{T}\left(t_{i}-d_{2}\left(t_{i}\right), t_{j+1}\right)
\end{array}\right]^{T}, \\
& x_{d m}=\left[\begin{array}{ll}
x^{T}\left(t_{i+1}, t_{j}-d_{1 m}\right) & x^{T}\left(t_{i}-d_{2 m}, t_{j+1}\right)
\end{array}\right]^{T} \text {, } \\
& x_{d M}=\left[\begin{array}{ll}
x^{T}\left(t_{i+1}, t_{j}-d_{1 M}\right) & x^{T}\left(t_{i}-d_{2 M}, t_{j+1}\right)
\end{array}\right]^{T}, \\
& n_{1 M} \mathrm{~T}_{v}=d_{1 M}, \quad n_{2 M} \mathrm{~T}_{h}=d_{2 M}, \quad n_{1 m} \mathrm{~T}_{v}=d_{1 m}, \\
& n_{2 m} \mathrm{~T}_{h}=d_{2 m}, \quad d_{12}=d_{1 M}-d_{1 m}, \quad d_{21}=d_{2 M}-d_{2 m} .
\end{aligned}
$$

For matrices $M_{1}, M_{2}, N_{1}$, and $N_{2}$, one has that

$$
\begin{aligned}
& 0=2 \mathrm{~T}_{v} \xi_{2}^{(1) T} M_{1}\left[x\left(t_{i+1}, t_{j}-d_{1}\left(t_{j}\right)\right)-x\left(t_{i+1}, t_{j}-d_{1 M}\right)-\sum_{\beta=\frac{d_{1}\left(j_{j}\right)}{T_{v}}+1}^{n_{1 M}} \bar{y}^{T}\left(t_{i+1}, t_{j}-\beta \mathrm{T}_{v}\right)\right], \\
& 0=2 \mathrm{~T}_{h} \xi_{2}^{(2) T} M_{2}\left[x\left(t_{i}-d_{2}\left(t_{i}\right), t_{j+1}\right)-x\left(t_{i}-d_{2 M}, t_{j+1}\right)-\sum_{\beta=\frac{d_{2}\left(t_{j}\right)}{T_{h}}+1}^{n_{2 M}} \bar{y}^{T}\left(t_{i}-\beta \mathrm{T}_{h}, t_{j+1}\right)\right], \\
& 0=2 \mathrm{~T}_{\nu} \xi_{2}^{(1) T} N_{1}\left[x\left(t_{i+1}, t_{j}-d_{1 m}\right)-x\left(t_{i+1}, t_{j}-d_{1}\left(t_{j}\right)\right)-\sum_{\beta=n_{1 m}+1}^{\frac{d_{1}\left(t_{j}\right)}{T_{v}}} \bar{y}^{T}\left(t_{i+1}, t_{j}-\beta \mathrm{T}_{v}\right)\right],
\end{aligned}
$$




$$
0=2 \mathrm{~T}_{h} \xi_{2}^{(2) T} N_{2}\left[x\left(t_{i}-d_{2 m}, t_{j+1}\right)-x\left(t_{i}-d_{2}\left(t_{i}\right), t_{j+1}\right)-\sum_{\beta=n_{2 m}+1}^{\frac{d_{2}\left(t_{j}\right)}{T_{h}}} \bar{y}^{T}\left(t_{i}-\beta \mathrm{T}_{h}, t_{j+1}\right)\right],
$$

where

$$
\begin{array}{ll}
\xi_{2}^{(1)}=\left[\begin{array}{ll}
x^{T}\left(t_{i+1}, t_{j}-d_{1}\left(t_{j}\right)\right) & x^{T}\left(t_{i+1}, t_{j}-d_{1 m}\right)
\end{array}\right]^{T}, \\
\xi_{2}^{(2)}=\left[\begin{array}{ll}
x^{T}\left(t_{i}-d_{2}\left(t_{i}\right), t_{j+1}\right) & x^{T}\left(t_{i}-d_{2 m}, t_{j+1}\right)
\end{array}\right]^{T}, \\
M_{1}=\left[\begin{array}{ll}
M_{11}^{T} & M_{12}^{T}
\end{array}\right]^{T}, \quad M_{2}=\left[\begin{array}{ll}
M_{21}^{T} & M_{22}^{T}
\end{array}\right]^{T}, \\
N_{1}=\left[\begin{array}{ll}
N_{11}^{T} & N_{12}^{T}
\end{array}\right]^{T}, \quad N_{2}=\left[\begin{array}{ll}
N_{21}^{T} & N_{22}^{T}
\end{array}\right]^{T} .
\end{array}
$$

Using equalities (16)-(20), the following inequality is given:

$$
\begin{aligned}
& \delta V\left(x\left(t_{i}, t_{j}\right)\right) \\
& \leq \frac{\mathrm{T}_{v}}{d_{12}} \sum_{\beta=\frac{d_{1}\left(t_{j}\right)}{T_{v}}+1}^{n_{1 M}}\left[\begin{array}{c}
\xi_{1} \\
-d_{12} \bar{y}\left(t_{i+1}, t_{j}-\beta \mathrm{T}_{v}\right)
\end{array}\right]^{T}\left[\begin{array}{cc}
\Psi & \Psi_{11} \\
\Psi_{11}^{T} & -Z_{1}
\end{array}\right]\left[\begin{array}{c}
\xi_{1} \\
-d_{12} \bar{y}\left(t_{i+1}, t_{j}-\beta \mathrm{T}_{v}\right)
\end{array}\right] \\
& +\frac{\mathrm{T}_{v}}{d_{12}} \sum_{\beta=n_{1 m}+1}^{\frac{d_{1}\left(t_{j}\right)}{\mathrm{T}_{v}}}\left[\begin{array}{c}
\xi_{1} \\
-d_{12} \bar{y}\left(t_{i+1}, t_{j}-\beta \mathrm{T}_{v}\right)
\end{array}\right]^{T}\left[\begin{array}{cc}
\Psi & \Psi_{12} \\
\Psi_{12}^{T} & -Z_{1}
\end{array}\right]\left[\begin{array}{c}
\xi_{1} \\
-d_{12} \bar{y}\left(t_{i+1}, t_{j}-\beta \mathrm{T}_{v}\right)
\end{array}\right] \\
& +\frac{\mathrm{T}_{h}}{d_{21}} \sum_{\beta=\frac{d_{2}\left(t_{j}\right)}{\mathrm{T}_{h}}+1}^{n_{2 M}}\left[\begin{array}{c}
\xi_{1} \\
-d_{21} \bar{y}\left(t_{i}-\beta \mathrm{T}_{h}, t_{j+1}\right)
\end{array}\right]^{T}\left[\begin{array}{cc}
\Psi & \Psi_{21} \\
\Psi_{21}^{T} & -Z_{2}
\end{array}\right]\left[\begin{array}{c}
\xi_{1} \\
-d_{21} \bar{y}\left(t_{i}-\beta \mathrm{T}_{h}, t_{j+1}\right)
\end{array}\right] \\
& +\frac{\mathrm{T}_{h}}{d_{21}} \sum_{\beta=n_{2 m}+1}^{\frac{d_{2}\left(t_{j}\right)}{\mathrm{T}_{h}}}\left[\begin{array}{c}
\xi_{1} \\
-d_{21} \bar{y}\left(t_{i}-\beta \mathrm{T}_{h}, t_{j+1}\right)
\end{array}\right]^{T}\left[\begin{array}{cc}
\Psi & \Psi_{22} \\
\Psi_{22}^{T} & -Z_{2}
\end{array}\right]\left[\begin{array}{c}
\xi_{1} \\
-d_{21} \bar{y}\left(t_{i}-\beta \mathrm{T}_{h}, t_{j+1}\right)
\end{array}\right],
\end{aligned}
$$

where

$$
\Psi=\Psi_{1}+\Phi_{1}^{T} P \Phi_{1}+\Phi_{2}^{T}\left(d_{1 m}^{2} S_{1}+d_{12}^{2} Z_{1}\right) \Phi_{2}+\Phi_{3}^{T}\left(d_{2 m}^{2} S_{2}+d_{21}^{2} Z_{2}\right) \Phi_{3}
$$

Moreover, sufficient conditions for $\delta V\left(x\left(t_{i}, t_{j}\right)\right)<0$ are given as follows:

$$
\begin{aligned}
& {\left[\begin{array}{cc}
\Psi & \Psi_{11} \\
\Psi_{11}^{T} & -Z_{1}
\end{array}\right]<0, \quad\left[\begin{array}{cc}
\Psi & \Psi_{12} \\
\Psi_{12}^{T} & -Z_{1}
\end{array}\right]<0,} \\
& {\left[\begin{array}{cc}
\Psi & \Psi_{21} \\
\Psi_{21}^{T} & -Z_{2}
\end{array}\right]<0, \quad\left[\begin{array}{cc}
\Psi & \Psi_{22} \\
\Psi_{22}^{T} & -Z_{2}
\end{array}\right]<0 .}
\end{aligned}
$$

Using inequalities in (22) and Lemma 3, inequalities (10) and (11) are obtained. Using Schur's complements, inequalities (10) and (11) are converted to inequalities (14) and (15), respectively. If inequalities (14) and (15) hold, then one has that $\delta V\left(x\left(t_{i}, t_{j}\right)\right)<0$, which implies that the 2-D delta operator system (3) with $u\left(t_{i}, t_{j}\right)=0$ is asymptotically stable. The proof is completed. 


\subsection{Stabilization}

In the subsection, a sufficient stabilization condition is provided for the 2-D delta operator systems (7) with time-varying delays and actuator saturation.

Theorem 2 For given scalars $\tau_{1}, \tau_{2}, \tau_{3}, \tau_{4}, \tau_{5}, d_{1 m}, d_{2 m}, d_{1 M}$, and $d_{2 M}$, the 2-D delta operator system (7) is asymptotically stable if there exist matrices $\widetilde{X}, \widetilde{Y}, \widetilde{P}>0, \widetilde{Q}>0, \widetilde{R}_{1}>0$, $\widetilde{R}_{2}>0, \widetilde{Q}_{1}>0, \widetilde{Q}_{2}>0, \widetilde{S}_{1}>0, \widetilde{S}_{2}>0, \widetilde{Z}_{1}>0, \widetilde{Z}_{2}>0, \widetilde{M}_{11}, \widetilde{M}_{12}, \widetilde{M}_{21}, \widetilde{M}_{22}, \widetilde{N}_{11}, \widetilde{N}_{12}, \widetilde{N}_{21}, \tilde{N}_{22}$, $V_{1}$, and $V_{2}$ such that the following LMIs hold:

$$
\begin{aligned}
& {\left[\begin{array}{cccc}
-\widetilde{Z}_{1} & \widetilde{\Psi}_{11} & \widetilde{X} & 0 \\
* & \widetilde{\Psi}_{1} & \widetilde{\Psi}_{12}^{T} & \widetilde{\Phi}^{T} \\
* & * & -\widetilde{Z}_{1} & 0 \\
* & * & * & \widetilde{\Psi}_{2}
\end{array}\right]<0,} \\
& {\left[\begin{array}{cccc}
-\widetilde{Z}_{2} & \widetilde{\Psi}_{21} & \widetilde{Y} & 0 \\
* & \widetilde{\Psi}_{1} & \widetilde{\Psi}_{22}^{T} & \widetilde{\Phi}^{T} \\
* & * & -\widetilde{Z}_{2} & 0 \\
* & * & * & \widetilde{\Psi}_{2}
\end{array}\right]<0,}
\end{aligned}
$$

and

$$
\Omega(P) \subset \mathcal{L}(H)
$$

where

$$
\begin{aligned}
& \widetilde{\Phi}^{T}=\left[\begin{array}{llllll}
\widetilde{\Phi}_{1}^{T} & \tau_{1} d_{1 m}^{2} \widetilde{\Phi}_{2}^{T} & \tau_{3} d_{12}^{2} \widetilde{\Phi}_{2}^{T} & \tau_{2} d_{2 m}^{2} \widetilde{\Phi}_{3}^{T} & \tau_{4} d_{21}^{2} \widetilde{\Phi}_{3}^{T}
\end{array}\right], \\
& \widetilde{\Psi}_{11}=\left[\begin{array}{llllllll}
0 & 0 & \tilde{M}_{11} & 0 & \tilde{M}_{12} & 0 & 0 & 0
\end{array}\right] \text {, } \\
& \widetilde{\Psi}_{12}=\left[\begin{array}{llllllll}
0 & 0 & \widetilde{N}_{11} & 0 & \widetilde{N}_{12} & 0 & 0 & 0
\end{array}\right] \text {, } \\
& \widetilde{\Psi}_{21}=\left[\begin{array}{llllllll}
0 & 0 & 0 & \widetilde{M}_{21} & 0 & \tilde{M}_{22} & 0 & 0
\end{array}\right] \text {, } \\
& \widetilde{\Psi}_{22}=\left[\begin{array}{llllllll}
0 & 0 & 0 & \tilde{N}_{21} & 0 & \tilde{N}_{22} & 0 & 0
\end{array}\right] \text {, } \\
& \widetilde{\Psi}_{1}=\left[\begin{array}{cccc}
-\bar{Q}_{1}+\widetilde{R}+\hat{Q}_{1}-\widetilde{S} & 0 & \widetilde{S} & 0 \\
* & -\tilde{Q}_{3}+\widehat{M}_{1}+\widehat{M}_{1}^{T}-\widehat{N}_{1}-\widehat{N}_{1}^{T} & \widehat{N}_{1}+\widehat{M}_{2}^{T}-\widehat{N}_{2}^{T} & -\widehat{M}_{1} \\
* & * & -\widetilde{S}^{2} \widehat{N}_{2}+\widehat{N}_{2}^{T} & -\widehat{M}_{2} \\
* & * & * & -\widetilde{R}^{2}
\end{array}\right] \text {, } \\
& \widetilde{\Psi}_{2}=\operatorname{diag}\left\{-\widetilde{P},-\tau_{1} d_{1 m}^{2} \widetilde{P},-\tau_{3} d_{12}^{2} \widetilde{P},-\tau_{2} d_{2 m}^{2} \widetilde{P},-\tau_{4} d_{21}^{2} \widetilde{P}\right\}, \quad \tilde{Q}_{3}=\operatorname{diag}\left\{\mathrm{T}_{v} \widetilde{Q}_{1}, \mathrm{~T}_{h} \widetilde{Q}_{2}\right\}, \\
& \hat{Q}_{1}=\operatorname{diag}\left\{\left(d_{12}+\mathrm{T}_{v}\right) \widetilde{Q}_{1},\left(d_{21}+\mathrm{T}_{h}\right) \widetilde{Q}_{2}\right\}, \quad \bar{Q}_{1}=\operatorname{diag}\{\widetilde{Q}, \widetilde{P}-\widetilde{Q}\}, \quad \widetilde{R}=\operatorname{diag}\left\{\widetilde{R}_{1}, \widetilde{R}_{2}\right\}, \\
& \widetilde{\Phi}_{1}=\left[\frac{1}{2}\left(\mathrm{~T}_{v} \bar{A}_{1}+I\right) \widetilde{P}+\frac{1}{2} \mathrm{~T}_{v} \bar{B}_{1}\left(D_{p} V_{1}+D_{p}^{-} V_{2}\right) \quad \frac{1}{2}\left(\mathrm{~T}_{h} \bar{A}_{2}+I\right) \widetilde{P}+\frac{1}{2} \mathrm{~T}_{h} \bar{B}_{2}\left(D_{p} V_{1}+D_{p}^{-} V_{2}\right)\right. \\
& \left.\frac{1}{2} \mathrm{~T}_{v} \bar{A}_{1 d} \widetilde{P} \quad \frac{1}{2} \mathrm{~T}_{h} \bar{A}_{2 d} \widetilde{P} \quad 0 \quad 00 \quad 0 \quad 0\right] \text {, } \\
& \widetilde{\Phi}_{2}=\left[\frac{1}{2}\left(\mathrm{~T}_{v} \bar{A}_{1}-I\right) \widetilde{P}+\frac{1}{2} \mathrm{~T}_{v} \bar{B}_{1}\left(D_{p} V_{1}+D_{p}^{-} V_{2}\right) \quad \frac{1}{2}\left(\mathrm{~T}_{h} \bar{A}_{2}+I\right) \widetilde{P}+\frac{1}{2} \mathrm{~T}_{h} \bar{B}_{2}\left(D_{p} V_{1}+D_{p}^{-} V_{2}\right)\right. \\
& \left.\frac{1}{2} \mathrm{~T}_{v} \bar{A}_{1 d} \widetilde{P} \quad \frac{1}{2} \mathrm{~T}_{h} \bar{A}_{2 d} \widetilde{P} \quad 0 \quad 0 \quad 00 \quad 0\right] \text {, }
\end{aligned}
$$




$$
\begin{aligned}
& \widetilde{\Phi}_{3}=\left[\frac{1}{2}\left(\mathrm{~T}_{v} \bar{A}_{1}+I\right) \widetilde{P}+\frac{1}{2} \mathrm{~T}_{v} \bar{B}_{1}\left(D_{p} V_{1}+D_{p}^{-} V_{2}\right) \quad \frac{1}{2}\left(\mathrm{~T}_{h} \bar{A}_{2}-I\right) \widetilde{P}+\frac{1}{2} \mathrm{~T}_{h} \bar{B}_{2}\left(D_{p} V_{1}+D_{p}^{-} V_{2}\right)\right. \\
& \left.\frac{1}{2} \mathrm{~T}_{v} \bar{A}_{1 d} \widetilde{P} \quad \frac{1}{2} \mathrm{~T}_{h} \bar{A}_{2 d} \widetilde{P} \quad 0 \quad 00 \quad 0 \quad 0\right], \\
& \widetilde{S}=\operatorname{diag}\left\{\mathrm{T}_{v}^{2} \widetilde{S}_{1}, \mathrm{~T}_{h}^{2} \widetilde{S}_{2}\right\}, \quad S_{1}=\tau_{1} P, \quad S_{2}=\tau_{2} P, \\
& \widetilde{M}_{1}=\left[\begin{array}{ll}
\widetilde{M}_{11}^{T} & \tilde{M}_{12}^{T}
\end{array}\right]^{T}, \quad \widetilde{M}_{2}=\left[\begin{array}{ll}
\widetilde{M}_{21}^{T} & \widetilde{M}_{22}^{T}
\end{array}\right]^{T}, \\
& \tilde{N}_{1}=\left[\begin{array}{ll}
\widetilde{N}_{11}^{T} & \tilde{N}_{12}^{T}
\end{array}\right]^{T}, \quad Z_{1}=\tau_{3} P, \quad Z_{2}=\tau_{4} P, \quad \widetilde{N}_{2}=\left[\begin{array}{ll}
\tilde{N}_{21}^{T} & \tilde{N}_{22}^{T}
\end{array}\right]^{T}, \\
& \widehat{N}_{1}=\operatorname{diag}\left\{\mathrm{T}_{v} \widetilde{N}_{11}, \mathrm{~T}_{h} \widetilde{N}_{21}\right\}, \quad \widehat{N}_{2}=\operatorname{diag}\left\{\mathrm{T}_{v} \widetilde{N}_{12}, \mathrm{~T}_{h} \widetilde{N}_{22}\right\}, \quad Q=\tau_{5} P, \\
& \widehat{M}_{1}=\operatorname{diag}\left\{\mathrm{T}_{v} \widetilde{M}_{11}, \mathrm{~T}_{h} \widetilde{M}_{21}\right\}, \quad \widehat{M}_{2}=\operatorname{diag}\left\{\mathrm{T}_{v} \widetilde{M}_{12}, \mathrm{~T}_{h} \widetilde{M}_{22}\right\}, \quad p=1,2, \ldots, 2^{m} .
\end{aligned}
$$

Furthermore, an estimate of the domain of attraction for system (7) is given as $\Gamma\left(\eta_{1}, \eta_{2}\right.$, $\left.\eta_{3}, \eta_{4}\right) \leq 1$, where

$$
\begin{aligned}
\Gamma\left(\eta_{1}, \eta_{2}, \eta_{3}, \eta_{4}\right) \\
=\eta_{1}^{2}\left(\lambda_{\max }(Q)+d_{1 M} \lambda_{\max }\left(R_{1}\right)+\frac{d_{1 M}^{2}-d_{1 m}^{2}+\mathrm{T}_{v}\left(d_{1 M}+d_{1 m}\right)}{2} \lambda_{\max }\left(Q_{1}\right)\right) \\
\quad+\eta_{3}^{2}\left(\frac{d_{1 m}^{2}\left(\mathrm{~T}_{v}+d_{1 m}\right)}{2} \lambda_{\max }\left(S_{1}\right)+\mathrm{T}_{v}\left(d_{1 M}-d_{1 m}\right)^{2} \lambda_{\max }\left(Z_{1}\right)\right) \\
\quad+\eta_{2}^{2}\left(\lambda_{\max }(P-Q)+d_{2 M} \lambda_{\max }\left(R_{2}\right)+\frac{d_{2 M}^{2}-d_{2 m}^{2}+\mathrm{T}_{h}\left(d_{2 M}+d_{2 m}\right)}{2} \lambda_{\max }\left(Q_{2}\right)\right) \\
\quad+\eta_{4}^{2}\left(\frac{d_{2 m}^{2}\left(\mathrm{~T}_{h}+d_{2 m}\right)}{2} \lambda_{\max }\left(S_{2}\right)+\mathrm{T}_{h}\left(d_{2 M}-d_{2 m}\right)^{2} \lambda_{\max }\left(Z_{2}\right)\right) .
\end{aligned}
$$

Note that matrices $K$ and $H$ are given as follows:

$$
K=V_{1} \widetilde{P}^{-1}, \quad H=V_{2} \widetilde{P}^{-1}
$$

Proof Using Lemma 1, the 2-D delta operator system (7) is rewritten as

$$
\begin{aligned}
\delta x\left(t_{i+1}, t_{j+1}\right)= & \bar{A}_{1} x\left(t_{i+1}, t_{j}\right)+\bar{A}_{2} x\left(t_{i}, t_{j+1}\right)+\bar{A}_{1 d} x\left(t_{i+1}, t_{j}-d_{1}\left(t_{j}\right)\right)+\bar{A}_{2 d} x\left(t_{i}-d_{2}\left(t_{i}\right), t_{j+1}\right) \\
& +\bar{B}_{1} \sum_{p=1}^{2^{m}} \eta_{p}\left(D_{p} K+D_{p}^{-} H\right) x\left(t_{i+1}, t_{j}\right)+\bar{B}_{2} \sum_{p=1}^{2^{m}} \eta_{p}\left(D_{p} K+D_{p}^{-} H\right) x\left(t_{i}, t_{j+1}\right) \\
& =\sum_{p=1}^{2^{m}} \eta_{p}\left(\bar{A}_{1}+\bar{B}_{1}\left(D_{p} K+D_{p}^{-} H\right)\right) x\left(t_{i+1}, t_{j}\right)+\bar{A}_{1 d} x\left(t_{i+1}, t_{j}-d_{1}\left(t_{j}\right)\right) \\
& +\sum_{p=1}^{2^{m}} \eta_{p}\left(\bar{A}_{2}+\bar{B}_{2}\left(D_{p} K+D_{p}^{-} H\right)\right) x\left(t_{i}, t_{j+1}\right)+\bar{A}_{2 d} x\left(t_{i}-d_{2}\left(t_{i}\right), t_{j+1}\right)
\end{aligned}
$$

In Theorem 1, parameters $\bar{A}_{1}$ and $\bar{A}_{2}$ are replaced by

$$
\sum_{p=1}^{2^{m}} \eta_{p}\left(\bar{A}_{1}+\bar{B}_{1}\left(D_{p} K+D_{p}^{-} H\right)\right)
$$


and

$$
\sum_{p=1}^{2^{m}} \eta_{p}\left(\bar{A}_{2}+\bar{B}_{2}\left(D_{p} K+D_{p}^{-} H\right)\right)
$$

respectively. Pre-multiplying and post-multiplying inequalities with input saturation by a diagonal matrix

$$
\operatorname{diag}\{\widetilde{P}, \widetilde{P}, \widetilde{P}, \widetilde{P}, \widetilde{P}, \widetilde{P}, \widetilde{P}, \widetilde{P}, \widetilde{P}, \widetilde{P}, \widetilde{P}, \widetilde{P}, \widetilde{P}, \widetilde{P}, \widetilde{P}, \widetilde{P}\}
$$

and letting

$$
\begin{aligned}
& \widetilde{P}=P^{-1}, \quad \widetilde{Q}=\widetilde{P} Q \widetilde{P}, \quad \widetilde{R}_{1}=\widetilde{P} R_{1} \widetilde{P}, \quad \widetilde{R}_{2}=\widetilde{P} R_{2} \widetilde{P}, \quad \widetilde{Q}_{1}=\widetilde{P} Q_{1} \widetilde{P}, \\
& \widetilde{Q}_{2}=\widetilde{P} Q_{2} \widetilde{P}, \quad \widetilde{S}_{2}=\widetilde{P} S_{2} \widetilde{P}, \quad \widetilde{Z}_{1}=\widetilde{P} Z_{1} \widetilde{P}, \quad \widetilde{Z}_{2}=\widetilde{P} Z_{2} \widetilde{P}, \quad \widetilde{M}_{11}=\widetilde{P} M_{11} \widetilde{P}, \\
& \widetilde{M}_{12}=\widetilde{P} M_{12} \widetilde{P}, \quad \widetilde{S}_{1}=\widetilde{P} S_{1} \widetilde{P}, \quad \widetilde{M}_{21}=\widetilde{P} M_{21} \widetilde{P}, \quad \widetilde{M}_{22}=\widetilde{P} M_{22} \widetilde{P}, \\
& \widetilde{N}_{11}=\widetilde{P} N_{11} \widetilde{P}, \quad \widetilde{N}_{12}=\widetilde{P} N_{12} \widetilde{P}, \quad \widetilde{N}_{21}=\widetilde{P} N_{21} \widetilde{P}, \quad \widetilde{N}_{22}=\widetilde{P} N_{22} \widetilde{P}, \\
& \widetilde{X}=\widetilde{P} X \widetilde{P}, \quad \widetilde{Y}=\widetilde{P} Y \widetilde{P}, \quad V_{1}=K \widetilde{P}, \quad V_{2}=H \widetilde{P},
\end{aligned}
$$

inequalities (23) and (24) are obtained.

For $\delta V\left(x\left(t_{i}, t_{j}\right)\right)<0$, one has that

$$
\begin{aligned}
& x^{T}\left(t_{i+1}, t_{j+1}\right) P x\left(t_{i+1}, t_{j+1}\right) \\
& \leq V\left(x\left(t_{i}, t_{j}\right)\right) \leq V\left(x_{0,0}\right) \\
& \leq \max _{\theta_{1} \in\left[-d_{1 M}, 0\right]}\left\|\phi_{v}\left(\theta_{1}\right)\right\|^{2} \\
& \times\left(\lambda_{\max }(Q)+d_{1 M} \lambda_{\max }\left(R_{1}\right)+\frac{d_{1 M}^{2}-d_{1 m}^{2}+\mathrm{T}_{v}\left(d_{1 M}+d_{1 m}\right)}{2} \lambda_{\max }\left(Q_{1}\right)\right) \\
&+\max _{\theta_{1} \in\left[-d_{1 M}, 0\right]}\left\|\delta \phi_{v}\left(\theta_{1}\right)\right\|^{2}\left(\frac{d_{1 m}^{2}\left(\mathrm{~T}_{v}+d_{1 m}\right)}{2} \lambda_{\max }\left(S_{1}\right)+\mathrm{T}_{v}\left(d_{1 M}-d_{1 m}\right)^{2} \lambda_{\max }\left(Z_{1}\right)\right) \\
&+\max _{\theta_{2} \in\left[-d_{2 M}, 0\right]}\left\|\phi_{h}\left(\theta_{2}\right)\right\|^{2} \\
& \times\left(\lambda_{\max }(P-Q)+d_{2 M} \lambda_{\max }\left(R_{2}\right)+\frac{d_{2 M}^{2}-d_{2 m}^{2}+\mathrm{T}_{h}\left(d_{2 M}+d_{2 m}\right)}{2} \lambda_{\max }\left(Q_{2}\right)\right) \\
&+\max _{\theta_{2} \in\left[-d_{2 M}, 0\right]}\left\|\delta \phi_{h}\left(\theta_{2}\right)\right\|^{2}\left(\frac{d_{2 m}^{2}\left(\mathrm{~T}_{h}+d_{2 m}\right)}{2} \lambda_{\max }\left(S_{2}\right)+\mathrm{T}_{h}\left(d_{2 M}-d_{2 m}\right)^{2} \lambda_{\max }\left(Z_{2}\right)\right) \\
&= \Gamma\left(\eta_{1}, \eta_{2}, \eta_{3}, \eta_{4}\right) \leq 1 .
\end{aligned}
$$

It is obtained that $x^{T}\left(t_{i+1}, t_{j+1}\right) P x\left(t_{i+1}, t_{j+1}\right) \leq 1$ and all system trajectories that start from $\Gamma\left(\phi_{1}, \phi_{2}, \delta \phi_{1}, \delta \phi_{2}\right) \leq 1$ will remain within $x^{T}\left(t_{i+1}, t_{j+1}\right) P x\left(t_{i+1}, t_{j+1}\right) \leq 1$. The proof is completed.

In order to obtain a maximal estimate of the domain of attraction, letting $\eta_{1}=\frac{1}{\varepsilon_{1}} \eta_{2}=$ $\frac{1}{\varepsilon_{2}} \eta_{3}=\frac{1}{\varepsilon_{3}} \eta_{4}$ and denoting $\mathcal{X}_{R}$ to be an ellipsoid, one has that $\mathcal{X}_{R}:=\left\{x\left(t_{i}, t_{j}\right) \in R^{n}:\right.$ 
$\left.x^{T}\left(t_{i}, t_{j}\right) R x\left(t_{i}, t_{j}\right) \leq 1\right\}$. An optimization problem is formulated as follows:

$$
\begin{aligned}
& \widetilde{P}, \widetilde{Q}_{,} \widetilde{R}_{1}, \widetilde{R}_{2}, \widetilde{Q}_{1}, \widetilde{Q}_{2}, \widetilde{S}_{1}, \widetilde{,}_{2}, \widetilde{Z}_{1}, \widetilde{Z}_{2}, \widetilde{X}, \widetilde{Y}, K, H \\
& \text { s.t. } \begin{cases}\text { (i) } & \eta_{1} \mathcal{X}_{R} \subset \Omega(P), \\
\text { (ii) } & \text { Inequality (23), } \\
\text { (iii) } & \text { Inequality (24), } \\
\text { (iv) } & \Omega(P) \subset \mathcal{L}(H) .\end{cases}
\end{aligned}
$$

In the optimization problem (27), condition (iv) is translated into

$$
x^{T}\left(t_{i}, t_{j}\right) h_{q}^{T} h_{q} x\left(t_{i}, t_{j}\right) \leq x^{T}\left(t_{i}, t_{j}\right) P x\left(t_{i}, t_{j}\right), \quad \forall x\left(t_{i}, t_{j}\right) \neq 0 .
$$

Inequality (28) is equivalent to

$$
h_{q}^{T} h_{q}-P \leq 0 .
$$

Using Schur's complements, it is obtained that

$$
\left[\begin{array}{cc}
-P & h_{q}^{T} \\
* & -1
\end{array}\right] \leq 0 .
$$

Pre-multiplying and post-multiplying inequality (29) by a diagonal matrix $\operatorname{diag}\{\widetilde{P}, 1\}$, one has that

$$
\left[\begin{array}{cc}
-\widetilde{P} & Z \\
* & -1
\end{array}\right] \leq 0,
$$

where $Z=\widetilde{P} h_{q}^{T}$.

The optimization problem (27) is transformed into the following optimization problem:

$$
\begin{aligned}
& \min _{\widetilde{P}, \widetilde{Q}_{1}, \widetilde{R}_{1}, \widetilde{R}_{2}, \widetilde{Q}_{1}, \widetilde{\widetilde{Q}}_{2}, \widetilde{S}_{1}, \widetilde{S}_{2}, \widetilde{Z}_{1}, \widetilde{Z}_{2}, \widetilde{X}, \widetilde{Y}}{ }^{r} \\
& \text { s.t. } \begin{cases}\text { (i) } & \text { Inequality }(23), \\
\text { (ii) } & \text { Inequality }(24), \\
\text { (iii) } & {\left[\begin{array}{r}
-\widetilde{P} Z \\
*-1
\end{array}\right] 0,} \\
\text { (iv) } & \omega_{1} I-\widetilde{Q} \geq 0, \\
\text { (v) } & \left.\omega_{2} I-\widetilde{P}-\widetilde{Q}\right) \geq 0, \\
\text { (vi) } & \omega_{3} I-\widetilde{R_{1}} \geq 0, \\
\text { (vii) } & \omega_{4} I-\widetilde{R_{2}} \geq 0, \\
\text { viii) } & \omega_{5} I-\widetilde{Q_{1}} \geq 0, \\
\text { (ix) } & \omega_{6} I-\widetilde{Q_{2}} \geq 0, \\
\text { (x) } & \omega_{7} I-\widetilde{S_{1}} \geq 0, \\
\text { (xi) } & \omega_{8} I-\widetilde{S_{2}} \geq 0, \\
\text { (xii) } & \omega_{9} I-\widetilde{Z_{1}} \geq 0, \\
\text { (xiii) } & \omega_{10} I-\widetilde{Z_{2}} \geq 0,\end{cases}
\end{aligned}
$$


where

$$
\begin{aligned}
r= & \omega_{1}+d_{1 M} \omega_{3}+\frac{d_{1 M}^{2}-d_{1 m}^{2}+\mathrm{T}_{v}\left(d_{1 M}+d_{1 m}\right)}{2} \omega_{5}+\frac{d_{1 m}^{2}\left(\mathrm{~T}_{v}+d_{1 m}\right)}{2} \omega_{7} \\
& +\mathrm{T}_{\nu}\left(d_{1 M}-d_{1 m}\right)^{2} \omega_{9}+\omega_{2}+d_{2 M} \omega_{4}+\frac{d_{2 M}^{2}-d_{2 m}^{2}+\mathrm{T}_{h}\left(d_{2 M}+d_{2 m}\right)}{2} \omega_{6} \\
& +\frac{d_{2 m}^{2}\left(\mathrm{~T}_{h}+d_{2 m}\right)}{2} \omega_{8}+\mathrm{T}_{h}\left(d_{2 M}-d_{2 m}\right)^{2} \omega_{10} .
\end{aligned}
$$

Furthermore, a maximal estimate of the domain of attraction is obtained by $\eta_{1 \max }=\frac{1}{\sqrt{\Lambda}}$, where

$$
\begin{aligned}
\Lambda= & \lambda_{\max }(Q)+d_{1 M} \lambda_{\max }\left(R_{1}\right)+\frac{d_{1 M}^{2}-d_{1 m}^{2}+\mathrm{T}_{v}\left(d_{1 M}+d_{1 m}\right)}{2} \lambda_{\max }\left(Q_{1}\right) \\
& +\varepsilon_{2}\left(\frac{d_{1 m}^{2}\left(\mathrm{~T}_{v}+d_{1 m}\right)}{2} \lambda_{\max }\left(S_{1}\right)+\mathrm{T}_{v}\left(d_{1 M}-d_{1 m}\right)^{2} \lambda_{\max }\left(Z_{1}\right)+\lambda_{\max }(P-Q)\right) \\
& +\varepsilon_{1}\left(d_{2 M} \lambda_{\max }\left(R_{2}\right)+\frac{d_{2 M}^{2}-d_{2 m}^{2}+\mathrm{T}_{h}\left(d_{2 M}+d_{2 m}\right)}{2} \lambda_{\max }\left(Q_{2}\right)\right) \\
& +\varepsilon_{3}\left(\frac{d_{2 m}^{2}\left(\mathrm{~T}_{h}+d_{2 m}\right)}{2} \lambda_{\max }\left(S_{2}\right)+\mathrm{T}_{h}\left(d_{2 M}-d_{2 m}\right)^{2} \lambda_{\max }\left(Z_{2}\right)\right) .
\end{aligned}
$$

\section{Numerical examples}

In this section, two numerical examples are provided to illustrate the effectiveness of the developed techniques.

Example 1 A thermal process is expressed into a 2-D discrete-time FM second model with time delays in [2]. In the 2-D discrete-time FM second model, parameters are given as follows:

$$
A_{1}=\left[\begin{array}{ll}
0 & 1 \\
0 & 0
\end{array}\right], \quad A_{2}=\left[\begin{array}{cc}
0 & 0 \\
0.25 & 0.65
\end{array}\right], \quad A_{1 d}=\left[\begin{array}{ll}
0 & 0 \\
0 & 0
\end{array}\right], \quad A_{2 d}=\left[\begin{array}{cc}
0 & 0 \\
0 & -0.12
\end{array}\right] .
$$

In this example, a 2-D delta operator system with $u\left(t_{i}, t_{j}\right)=0$ is given as

$$
\begin{aligned}
\delta x\left(t_{i+1}, t_{j+1}\right)= & \bar{A}_{1} x\left(t_{i+1}, t_{j}\right)+\bar{A}_{1 d} x\left(t_{i+1}, t_{j}-d_{1}\left(t_{j}\right)\right) \\
& +\bar{A}_{2} x\left(t_{i}, t_{j+1}\right)+\bar{A}_{2 d} x\left(t_{i}-d_{2}\left(t_{i}\right), t_{j+1}\right),
\end{aligned}
$$

where

$$
\bar{A}_{1}=\frac{2 A_{1}-I}{\mathrm{~T}_{v}}, \quad \bar{A}_{1 d}=\frac{2 A_{1 d}}{\mathrm{~T}_{v}}, \quad \bar{A}_{2}=\frac{2 A_{2}-I}{\mathrm{~T}_{h}}, \quad \bar{A}_{2 d}=\frac{2 A_{2 d}}{\mathrm{~T}_{h}} .
$$

Sampling periods of system (32) are chosen as $\mathrm{T}_{v}=0.1$ and $\mathrm{T}_{h}=0.02$. Trajectories of two state variables for the 2-D delta operator system (32) with a time-delay upper bound $d_{1 M}=$ 24 are shown in Fig. 1. It is seen clearly from Fig. 1 that state responses converge to origin, which means that system (32) with a time-delay upper bound $d_{1 M}=24$ is asymptotically stable. 


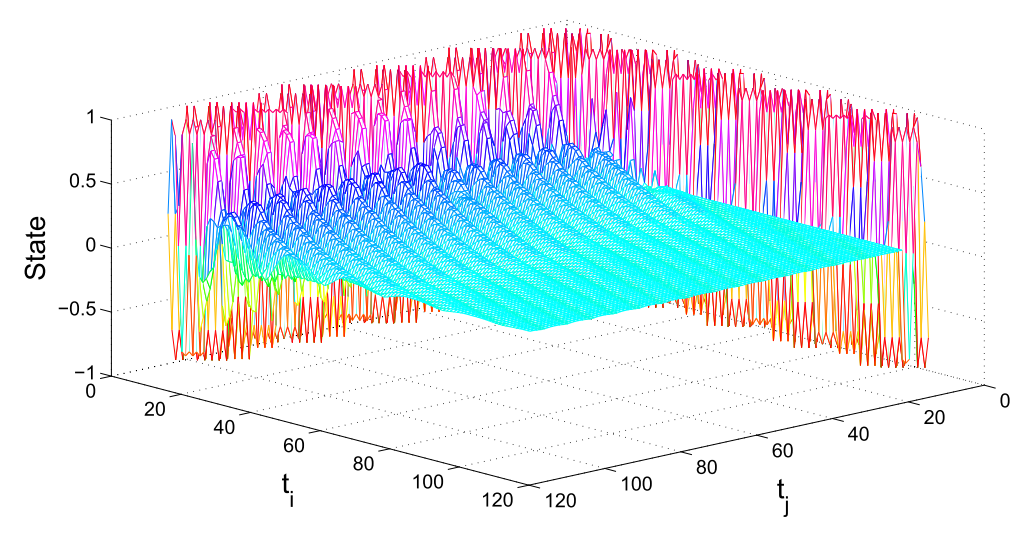

Figure 1 State trajectories of system (32) with a time-delay upper bound $d_{1 M}=24$

Table 1 Allowable time-delay upper bounds $d_{1 M}$

\begin{tabular}{lllll}
\hline & $d_{1 m}$ & $d_{1 M}$ & $d_{2 m}$ & $d_{2 M}$ \\
\hline$[2]$ & 1 & 13 & 1 & 11 \\
{$[10]$} & 1 & 20 & 1 & 11 \\
{$[12]$} & 1 & 20 & 1 & 11 \\
Theorem 1 & 1 & 24 & 1 & 11 \\
\hline
\end{tabular}

In this example, the 2-D delta operator system (32) with $1<d_{1}\left(t_{j}\right)<24$ is asymptotically stable. However, systems in [2,10], and [12] are asymptotically stable for $1<d_{1}\left(t_{j}\right)<13$, $1<d_{1}\left(t_{j}\right)<20$, and $1<d_{1}\left(t_{j}\right)<20$, respectively. Time-delay upper bounds $d_{1 M}$ given in [2, $10,12]$, and Theorem 1 in this paper are compared in Table 1 . Obviously, the time-delay upper bound $d_{1 M}$ provided in this paper is larger than the ones obtained in $[2,10]$, and [12].

Moreover, it is worth noting that the total number of decision variables in [2] and [10] are $16 n^{2}+6 n$ and $26 n^{2}+10 n$, respectively, while in Theorem 1 of this paper, they are $15 n^{2}+5 n$. It is obtained that a lower number of decision variables are needed in this paper than [2] and [10] for the asymptotic stability of the 2-D delta operator system (32). Therefore, the approach in this paper reduces the burden of numerical computation.

Example 2 A 2-D delta operator system with time-varying delays and actuator saturation is given as

$$
\begin{aligned}
\delta x\left(t_{i+1}, t_{j+1}\right)= & \bar{A}_{1} x\left(t_{i+1}, t_{j}\right)+\bar{A}_{1 d} x\left(t_{i+1}, t_{j}-d_{1}\left(t_{j}\right)\right)+\bar{B}_{1} \operatorname{sat}\left(u\left(t_{i+1}, t_{j}\right)\right) \\
& +\bar{A}_{2} x\left(t_{i}, t_{j+1}\right)+\bar{A}_{2 d} x\left(t_{i}-d_{2}\left(t_{i}\right), t_{j+1}\right)+\bar{B}_{2} \operatorname{sat}\left(u\left(t_{i}, t_{j+1}\right)\right),
\end{aligned}
$$

where

$$
\begin{array}{lll}
\bar{A}_{1}=\frac{2 A_{1}-I}{\mathrm{~T}_{v}}, & \bar{A}_{1 d}=\frac{2 A_{1 d}}{\mathrm{~T}_{v}}, & \bar{B}_{1}=\frac{2 B_{1}}{\mathrm{~T}_{v}}, \\
\bar{A}_{2}=\frac{2 A_{2}-I}{\mathrm{~T}_{h}}, & \bar{A}_{2 d}=\frac{2 A_{2 d}}{\mathrm{~T}_{h}}, & \bar{B}_{2}=\frac{2 B_{2}}{\mathrm{~T}_{h}},
\end{array}
$$




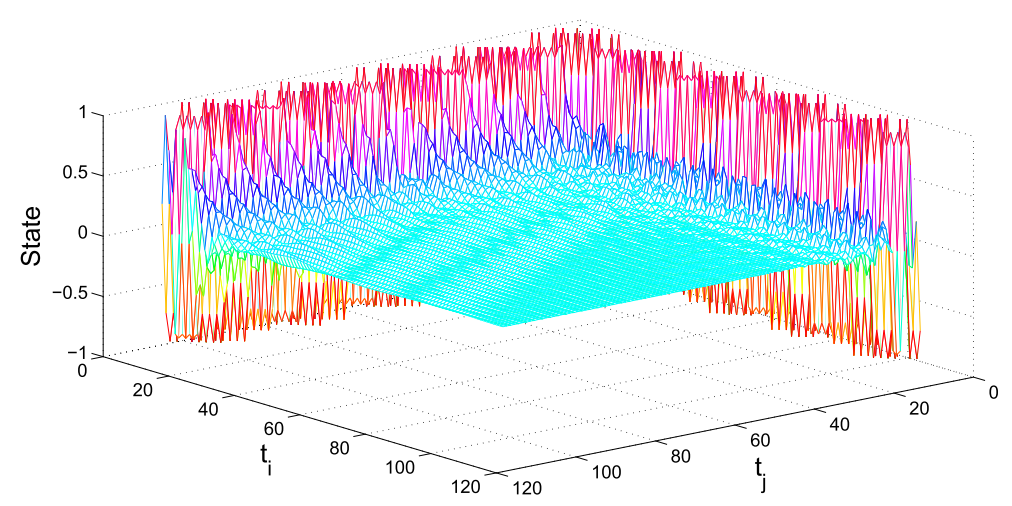

Figure 2 State trajectories of system (33) with a feedback gain matrix $K$

with

$$
\begin{array}{lll}
A_{1}=\left[\begin{array}{cc}
0.5 & -0.6 \\
0.1 & 0.2
\end{array}\right], & A_{1 d}=\left[\begin{array}{cc}
0.1 & 0 \\
0 & 0.2
\end{array}\right], & B_{1}=\left[\begin{array}{cc}
-1.5 & 0 \\
-1.1 & 0
\end{array}\right], \\
A_{2}=\left[\begin{array}{cc}
0.2 & 0 \\
0.5 & 0.1
\end{array}\right], & A_{2 d}=\left[\begin{array}{cc}
0 & 0 \\
0 & -0.01
\end{array}\right], & B_{2}=\left[\begin{array}{cc}
1.4 & 0.3 \\
0.7 & 0
\end{array}\right] .
\end{array}
$$

Time-varying delays of system (33) are satisfied with $1<d_{1}\left(t_{j}\right)<24$ and $1<d_{2}\left(t_{i}\right)<11$. Sampling periods of system (33) are chosen as $\mathrm{T}_{v}=0.1$ and $\mathrm{T}_{h}=0.02$. Letting parameters $\tau_{1}=0.01, \tau_{2}=0.02, \tau_{3}=0.03, \tau_{4}=0.03$, and $\tau_{5}=0.95$. By solving the optimization problem (30), matrices $K$ and $H$ are obtained as

$$
K=\left[\begin{array}{cc}
-0.0956 & -0.2842 \\
-0.4515 & 1.2239
\end{array}\right], \quad H=\left[\begin{array}{cc}
-0.0958 & -0.2818 \\
-0.4481 & 1.2158
\end{array}\right]
$$

Trajectories of two state variables for the 2-D delta operator system (33) are shown in Fig. 2. It is seen clearly from Fig. 2 that state responses converge to origin which means that system (33) is asymptotically stable with a feedback gain matrix $K$ given in equality (34).

Moreover, an estimate of the domain of attraction is shown in Fig. 3. The domain of attraction is a subset of state space and all system trajectories that start from the subset will eventually tend to origin. In Fig. 3, an estimate of the domain of attraction is shown for 2-D delta operator systems with time-varying delays and actuator saturation.

\section{Conclusion}

In this paper, the stabilization problem has been shown for the 2-D delta operator system with time-varying delays and actuator saturation. Free weighting matrices, 2-D Jensen inequalities, and LMIs approaches have been applied for stabilization analysis. Furthermore, the estimate of the domain of attraction has been proposed for the 2-D delta operator system. The state feedback controller has been designed by the Lyapunov-Krasovskii methods. Two numerical examples have been shown to illustrate the effectiveness and advantages of the developed techniques. Delay and fractional models are two different 


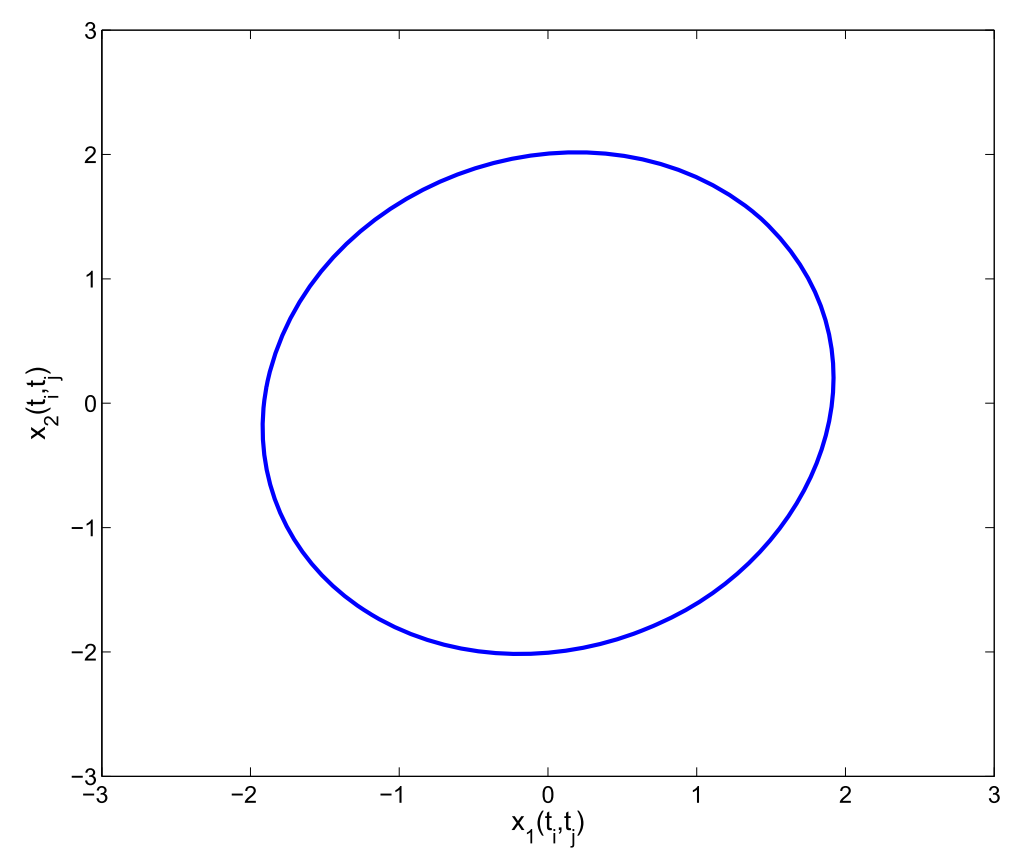

Figure 3 An estimate of the domain of attraction

memory models, fractional calculus has memory effects to depict the long-term behavior [27-29]. Fractional models can provide powerful tools to describe the hereditary and memory properties of different substances [30]. Meanwhile, time-delay optimal control problems have attracted wide attention in recent years [31,32]. All these will contribute to our research for 2-D delta operator systems in the future.

\section{Acknowledgements}

The authors would like to thank the anonymous reviewers for their detailed comments which helped to improve the quality of the paper.

\section{Funding}

This work was partially supported by the Natural Science Foundation of Hebei Province (F2018203370, F2016203467), National Natural Science Foundation of China (61673335).

\section{Availability of data and materials}

Not applicable.

\section{Competing interests}

The authors declare that they have no competing interests.

\section{Authors' contributions}

DP participated in the design of controller and stability analysis for 2-D systems with time-varying delays. TZ participated in the stabilization analysis for 2-D delta operator systems with time-varying delays and actuator saturation and drafted the manuscript. HY participated in the stability analysis for delta operator systems with actuator saturation. All authors read and approved the final manuscript.

\section{Authors' information}

Dan Peng is an associate professor of the School of Science, Yanshan University, Qinhuangdao, China. Her research interests include time-delay systems, nonlinear systems and 2-D systems. Ting Zhang is a master's graduate student of the School of Science, Yanshan University, Qinhuangdao, China. Her research interests include 2-D systems and delta operator systems. Hongjiu Yang is an associate professor of the Institute of Electrical Engineering, Yanshan University, Qinhuangdao, China. His research interests include delta operator systems and networked control systems.

\section{Author details}

${ }^{1}$ School of Science, Yanshan University, Qinhuangdao, China. ${ }^{2}$ Institute of Electrical Engineering, Yanshan University, Qinhuangdao, China. 


\section{Publisher's Note}

Springer Nature remains neutral with regard to jurisdictional claims in published maps and institutional affiliations.

\section{Received: 16 July 2018 Accepted: 15 October 2018 Published online: 28 November 2018}

\section{References}

1. Ghous, l., Xiang, Z.: Robust state feedback $H_{\infty}$ control for uncertain 2-D continuous state delayed systems in Roesser model. Multidimens. Syst. Signal Process. 27(2), 297-319 (2016)

2. Feng, Z., Xu, L., Wu, M., He, Y.: Delay-dependent robust stability and stabilisation of uncertain two-dimensional discrete systems with time-varying delays. IET Control Theory Appl. 4(10), 1959-1971 (2010)

3. Paszke, W., Galkowski, K., Rogers, E., Owens, D.: Linear repetitive process control theory applied to a physical example. Int. J. Appl. Math. Comput. Sci. 13(1), 87-99 (2003)

4. Huang, S., Xiang, Z: Stability and $I_{2}$-gain analysis for $2 \mathrm{D}$ discrete switched systems with time-varying delays in the second FM model. Adv. Differ. Equ. 2013, 56 (2013)

5. Wu, L., Yang, R., Shi, P., Su, X.: Stability analysis and stabilization of 2-D switched systems under arbitrary and restricted switchings. Automatica 59, 206-215 (2015)

6. Peng, D., Guan, X.: Output feedback $H_{\infty}$ control for 2-D state-delayed systems. Circuits Syst. Signal Process. 28(1), 147-167 (2009)

7. Peng, D., Guan, X:: $H_{\infty}$ filtering of 2-D discrete state-delayed systems. Multidimens. Syst. Signal Process. 20(3), 265-284 (2008)

8. Luo, Y., Wang, Z., Liang, J.: $H_{\infty}$ control for 2-D fuzzy systems with interval time-varying delays and missing measurements. IEEE Trans. Cybern. 47(2), 365-377 (2017)

9. Ghous, I., Xiang, Z., Karimi, H.: State feedback $H_{\infty}$ control for 2-D switched delay systems with actuator saturation in the second FM model. Circuits Syst. Signal Process., 34(7), 2167-2192 (2015)

10. Peng, D., Hua, C.: Delay-dependent stability and static output feedback control of 2-D discrete systems with interval time-varying delays. Asian J. Control 16(6), 1-9 (2014)

11. Peng, D., Hua, C.: Improved approach to delay-dependent stability and stabilisation of two-dimensional discrete-time systems with interval time-varying delays. IET Control Theory Appl. 9(12), 1839-1845 (2015)

12. Peng, D., Zhang, J., Hua, C., Gao, C.: A delay-partitioning approach to the stability analysis of 2-D linear discrete-time systems with interval time-varying delays. Int. J. Control. Autom. Syst. 16(2), 682-688 (2018)

13. Yuan, Y., Sun, F:: Delay-dependent stability criteria for time-varying delay neural networks in the delta domain Neurocomputing 125(11), 17-21 (2013)

14. Goodwin, G., Leal, R., Mayne, D., Middleton, R.: Rapprochement between continuous and discrete model reference adaptive control. Automatica 22(2), 199-207 (1986)

15. Yang, H., Li, X., Liu, Z., Hua, C.: Fault detection for uncertain fuzzy systems based on the delta operator approach Circuits Syst. Signal Process. 33(3), $733-759$ (2014)

16. Zhou, Q., Shao, X., Karimi, H., Zhu, J.: Stability of genetic regulatory networks with time-varying delay: delta operator method. Neurocomputing 149(3), 490-495 (2015)

17. Ran, M., Wang, Q., Dorg, C.: Stabilisation of a class of nonlinear systems with actuator saturation via active disturbance rejection control. Automatica 63, 302-310 (2016)

18. Guan, W., Liu, F.: Finite-time $H_{\infty}$ memory state feedback control for uncertain singular TS fuzzy time-delay system under actuator saturation. Adv. Differ. Equ. 2016, 52 (2016)

19. Ma, Y., Jing, Y.: Robust synchronization of chaotic systems with input saturation and time-varying delay. Adv. Differ. Equ. 2014, 124 (2014)

20. Hu, T., Lin, Z:: Control Systems with Actuator Saturation: Analysis and Design. Birkhaüser, Boston (2001)

21. Zhang, L., Boukas, E., Haidar, A.: Delay-range-dependent control synthesis for time-delay systems with actuator saturation. Automatica 44, 2691-2695 (2008)

22. Mao, Z., Jiang, B., Shi, P.: Fault-tolerant control for a class of nonlinear sampled-data systems via a Euler approximate observer. Automatica 46(11), 1852-1859 (2010)

23. Gu, K.: Partial solution of LMI in stability problem of time-delay systems. Proc. IEEE Conf. Decis. Control 1(1), 227-232 (1999)

24. Eielsen, A., Teo, Y., Fleming, A.: Improving robustness filter bandwidth in repetitive control by considering model mismatch. Asian J. Control 19(4), 1-11 (2016)

25. Zhou, L., She, J., Zhou, S.: A 2D system approach to the design of a robust modified repetitive-control system with a dynamic output-feedback controller. Int. J. Appl. Math. Comput. Sci. 24(2), 325-334 (2014)

26. Emelianov, M., Pakshin, V., Kowski, K.: Stabilization of differential repetitive processes. Autom. Remote Control 76(5), 786-800 (2015)

27. Wu, G., Baleanu, D.: Stability analysis of impulsive fractional difference equations. Fract. Calc. Appl. Anal. 21(2), 354-375 (2018)

28. Wu, G., Baleanu, D., Zeng, S.: Finite-time stability of discrete fractional delay systems: Gronwall inequality and stability criterion. Commun. Nonlinear Sci. Numer. Simul. 57, 299-308 (2018)

29. Wu, G., Baleanu, D., Xie, H., Chen, F.: Chaos synchronization of fractional chaotic maps based on stability results. Physica A 460, 374-383 (2016)

30. Hajipour, A., Hajipour, M., Baleanu, D.: On the adaptive sliding mode controller for a hyperchaotic fractional-order financial system. Physica A 497, 139-153 (2018)

31. Hajipour, M., Jajarmi, A., Baleanu, D.: An efficient nonstandard finite difference scheme for a class of fractional chaotic systems. J. Comput. Nonlinear Dyn. 13(2), 021013 (2018)

32. Jajarmi, A., Hajipour, M.: An efficient finite difference method for the time-delay optimal control problems with time-varying delay. Asian J. Control 19(2), 554-563 (2017) 